Document downloaded from:

http://hdl.handle.net/10251/101675

This paper must be cited as:

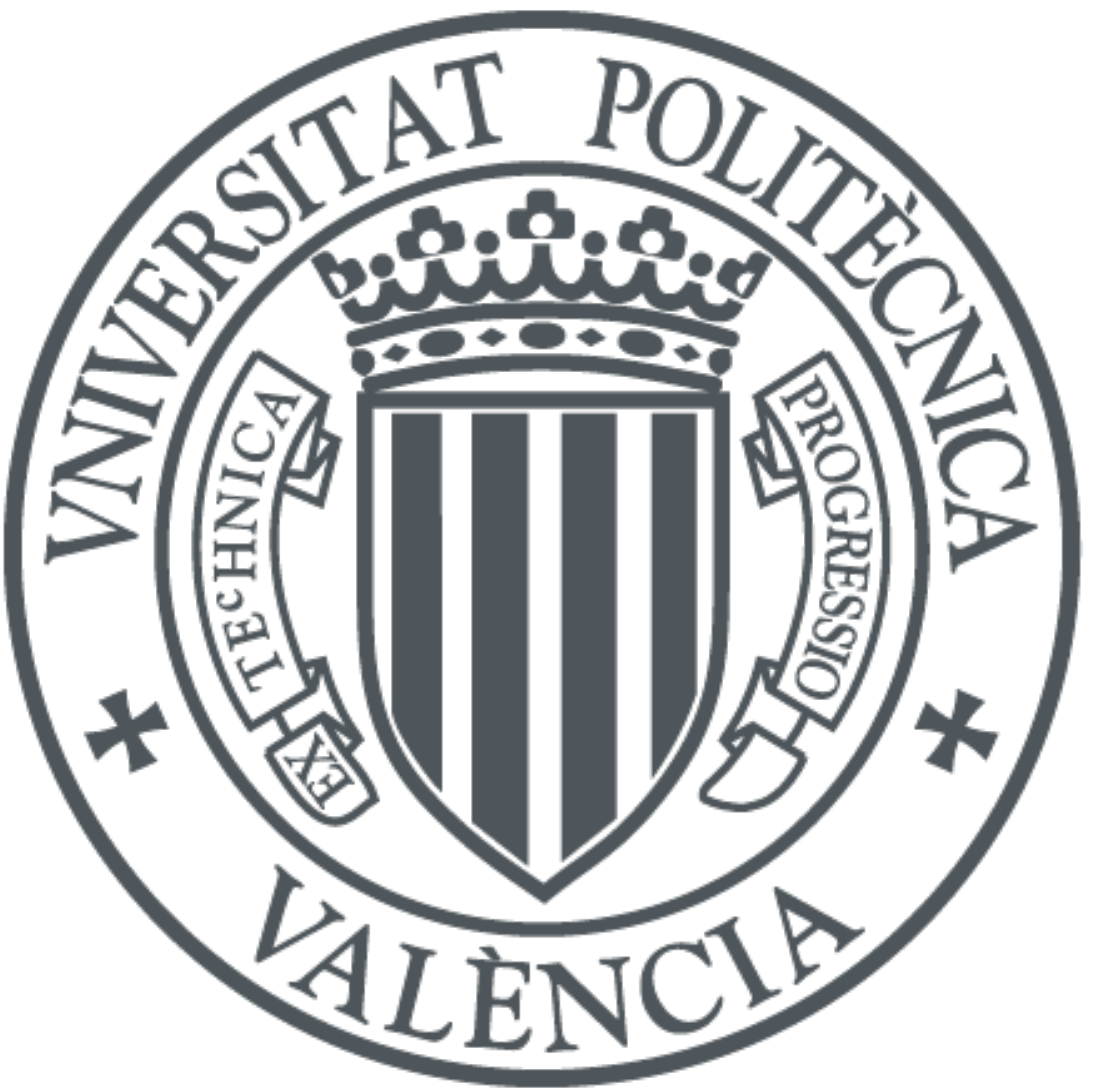

The final publication is available at

https://doi.org/10.1002/dac.3335

Copyright John Wiley \& Sons

Additional Information 


\title{
Design and Deployment of a Smart System for Data Gathering in Aquaculture Tanks Using Wireless Sensor Networks
}

\author{
Lorena Parra $^{1}$, Sandra Sendra ${ }^{1,2}$, Jaime Lloret ${ }^{1}$, Joel J.P.C. Rodrigues ${ }^{3,4,5}$ \\ ${ }^{1}$ Instituto de Investigación para la Gestión Integrada de zonas Costeras. Universitat Politècnica de \\ València, Spain \\ ${ }^{2}$ Signal Theory, Telematics and Communications Department, Universidad de Granada. Granada, \\ Spain \\ ${ }^{3}$ National Institute of Telecommunications (Inatel), CEP 37540-000. Santa Rita do Sapucaí, Minas \\ Gerais, Brazil \\ ${ }^{4}$ Instituto de Telecomunicações, University of Beira Interior, Covilhã, Portugal \\ ${ }^{5}$ ITMO University, 49 Kronverksky Pr., St. Petersburg, 197101, Russia.
}

Email: loparbo@doctor.upv.es, ssendra@ugr.es, jlloret@dcom.upv.es, joeljr@ieee.org

\begin{abstract}
The design of monitoring systems for marine areas has increased in the last years. One of the many advantages of Wireless Sensor Networks (WSNs) is the quick process in data acquisition. The information from sensors can be processed, stored and transmitted using protocols efficiently designed to energy saving and establishing the fastest routes. The processing and storing of data can be very useful for taking intelligent decisions for improving the water quality. The monitoring of water exchange in aquaculture tanks is very important to monitor the fish welfare. Thus, this paper presents the design, deployment and test of a smart data gathering system for monitoring several parameters in aquaculture tanks using a WSN. The system based on a server is able to request and collect data from several nodes and store them in a data base (DB). This information can be post-processed to take efficient decisions. The paper also presents the design of a conductivity sensor and a level sensor. These sensors are installed in several aquaculture tanks. The system was implemented using FlyPort modules. Finally, the data gathering system was tested in terms of .consumed bandwidth and the delay TCP packets delivering data from the sensors.
\end{abstract}

Index Terms - Smart System, Data Gathering, Wireless Sensor Network, FlyPort module, data acquisition system, conductivity sensor.

\section{INTRODUCTION}

Water quality has to be monitored continuously in aquaculture to ensure good management of the daily activity. The data gathered of the water quality facilitates to take different actions in order to prevent damages or to adapt the feeding process. However, the technology needed for it, is still in development or is expensive.

Wireless Sensor Networks (WSNs) are composed by small devices (nodes) that are able to sense data from the environment. The nodes have the capability to communicate between them. To collect data from the natural environments, these nodes should be sensors able to detect changes in physical and chemical magnitudes generated by events in the medium. These sensors should be capable of transforming these magnitudes into electric variables [1].

The fact of having multiple devices distributed along an area, communicating each other and sending information about the physicochemical parameters is very attractive for several environments. However, we should consider the drawbacks these mediums present, especially in underwater environments where the dispersive character of water worsen the data transmission [2]. Using those WSN, there is no need of going continuously to the place under study to take samples in order to analyze them at the laboratory. Comparing with traditional sampling and analysis procedure in chemical laboratory, WSNs offer several advantages. Besides the aforementioned displacements, the consumption of chemical reagents (which can be expensive and pollutant) and the destruction of the sample (precluding a repetition of the analysis) are some advantages. Other advantages are related to the real time monitoring and the possibility of taking intelligent decisions [3]. Using WSN there is no need to wait till the sample is carried to the laboratory in order to perform the analysis. For these reasons, the use of WSN is dramatically increasing. However, there are some disadvantages of using WSN related to the need of battery replacement. Some protocols have been developed to reduce the energy consumption of WSN $[4,5]$ and even energy harvesting 
techniques are applied as in radio networks [6]. There are several areas where WSNs are widely used for improving aspects of network architecture [7] and topology control [8]. This is the case of mobile networks [9] or cyber physical systems [10]. However, there are some other study areas where WSNs are used for monitoring systems in different environments such as food industry [11], investigation on volcanic eruptions [12], glaciers observation [13], precision agriculture animal and rural environment monitoring [14], among others, but in any case, the main aim of all these applications is to remotely monitor a facility located far from the monitoring centers using WSNs [15].

The majority of applications are implemented on land. But these nodes could be deployed in a changing medium and in that case we would need to locate the sensors [16, 17]. The use of those sensors in marine environments leads much more problems. Some WSN applications in marine environments are the following ones: pollution tracking [18], water quality monitoring [19] or precision aquaculture. The fish farms can be located at the sea or inland, but they always depend on the sea water. The quality of this water is crucial for the fish wellbeing, growth and survival. Some parameters like salinity can affect negatively to the fish performance. A decrease in the fish performance, growth or survival, impacts on the benefits of the fish farm. For this reason the monitoring on the water parameters is important for fish farms facilities. The nodes and sensors for underwater WSN must be waterproof, robust and have low energy recruitments or energy harvesting techniques. Those specifications usually increase the cost of the sensors and sensor nodes, thus increasing the investment of the fish farms. Fortunately, recently a new research line appears in the scientific community to obtain low cost sensors in order to spread the real use of WSN.

This paper presents the complete design, deployment and test of a smart system focused on precision aquaculture based on salinity sensors. The design, development and testing of a salinity sensor and water level sensor are shown, including its waterproof isolation. We also design the data gathering system of the wireless sensor nodes and how the data is stored into a database (DB). The node is capable of taking data from several sensors, although we only describe the salinity sensor. The rest of sensors included in our system were developed in previous work [19]. We also develop an algorithm to select and distribute the water along the aquaculture facility.

The structure of the rest of this paper is as follows. Section II presents several interesting previous works related to sensor development, data gathering systems to collect data from different environments and the effects of salinity in aquaculture. Section III presents our designed system and how it has been implemented. The architecture developed and the algorithm needed to control water levels and salinity concentration in the groups of fish tanks are shown in Section IV. Section V shows some tests related to the network performance. Finally, Section Vi presents the conclusion and future work.

\section{RELATED WORK}

This section shows the state-of-art related to this proposal. First, it is presented a collection of papers that show the effects of salinity on aquaculture fishes. Second, a survey on current salinity sensors is presented. Last part of this section presents several gathering data systems specially designed to be used inside WSNs are explained.

\section{A. Effects of salinity on fish}

In this subsection, different studies where authors evaluated the effects of the salinity in aquaculture fishes are shown. Authors evaluated the effects of salinity on their growth and survival at different development stages.

The effects of the salinity on the food consumption of fish are widely studied. V.C. Rubio et al. studied the effects of low salinities on juveniles of Dicentrarchus labrax [20] under low salinity conditions fishes. They observed that fishes reduced their food intake. R. Laiz-Carrión et al. [21] carried out similar studies with Sparus aurata and they observed that the maximum specific growth rate was reached at 12 ppt. L.A. Sampaio et al. [22] founded higher performances with juveniles of Paralichthys orbignyanus at higher salinity values. The effects of salinity on survival were also tested by different authors. T. I. J . Smith et al. [23] studied the effects on the eggs of Paralichthys lethostigma. The maximum percentaje of egg bouyancy appeared at higher salinities. The effects on eggs and larvae of Rachycentron canadum were studied by C. K. Faulk et al. in [24]. The best results were related to lowest salinities, from 0 to 3 ppt. A. Tandler et al. [25] found that the maximum survival for Sparus aurata was reached at lowest salinities. D. S. Field et al. [26] developed a test of Argyrosomus japonicus juveniles, where some individuals mantained at $0.6 \mathrm{ppt}$ presented stress sintoms and died. However, the letal effects on adults are not so common. Different studies were done in $[27,28]$ and no one found letal effects on adult fishes.

The performance of fish is highly affected by the salinity of the water. Thus, it is clear the need of salinity sensors for monitoring the water parameters where the fishes are growing. For a fish farm the obtention of high hatching rates and high growth rates are crutial to increase its benefits. There are two 
big groups of facilities; the ones in land where the fertilization is done, the eggs hatched and the larvaes growth; and the ones in the sea, where the juveniles growth until they have commertial size. The survival is the most important factor in the first one, those facilities use to take the water from the sea with pipes. They can exericice a minimum control on the water. The sensors are fundamental to monitor and correct the water parameters. The growth is the most imporant factor for the second one, those facilities have no control on the water parameters, they only can addapt their acctions. The sensors can bring informacion about the water parameters in real time and the workers can addapt the fish feed to the real need of the fish taking into account the environmental conditions.

\section{B. Salinity sensors}

In this subsection, several induction salinity sensors are introduced. Water density, conductivity and light refraction can be used to measure the water salinity [29, 30].

To measure the density in order to obtain the salinity of the water is not the best option for our application because the suspended particles that can be found in the aquaculture environment will affect the density value. Light refraction sensors [31, 32] are not an optimal solution. Those sensors have high maintenance requirements because they must be cleaned. The lens of this sensor can get dirty causing an underestimation or overestimation of salinity. The values of water conductivity can be easily related to salinity values.

There are two different techniques to measure the conductivity, the inductive and the conductive methodology. The selection of the technique depends on the characteristics of the environment where the sensor will be placed. The main difference between the inductive and conductive salinity sensors is the contact between the sensor and the water. In inductive sensors it is possible to isolate the physical part of the sensor form the environment using different covers. This isolation can prevent damages, reduces the needs of clean and makes easier the cleaning task. There are several inductive conductivity sensors in the related literature. The first paper found where the authors explore an inductive sensor was published in 1985 [33]. Only few papers show the design of an inductive sensor. In [34, 35], T. Pham et al. present the design, creation and evaluation of an inductive sensor. The sensor works at $250 \mathrm{kHz}[34]$ and $500 \mathrm{kHz}$ [35]. They designed and created the sensing part of the sensor, two toroidal coils. The sensor was calibrated from $25 \mathrm{mS} / \mathrm{cm}$ to $65 \mathrm{mS} / \mathrm{cm}$. A package for the sensor and a data logger were also created by the authors. However, the packaging technique, with epoxy material, was not optimal and the sensor did not work after packaging. R.T. Wood et al. designed a salinity sensor formed by two toroidal coils [36]. Authors used two pairs of coils for their test. The coils were powered with a $250 \mathrm{kHz}$ square wave. The first pair was calibrated from $4 \mathrm{mS} / \mathrm{cm}$ to $49 \mathrm{mS} / \mathrm{cm}$ and the second pair from 0.45 to $3.4 \mathrm{mS} / \mathrm{cm}$. L. Parra et al. [37] developed new salinity sensors. They used a solenoidal coil for generating the magnetic field and took the measures with a Hall Effect sensor. Authors were capable to take measurements from 0.0028 $\mathrm{mS} / \mathrm{cm}$ to $194 \mathrm{mS} / \mathrm{cm}$. Authors used 4 different coil combinations (toroidal and solenoidal coils) in order to find the best combination to optimize the sensor design. They found that different combinations brought different results and had different measurable ranges. The calibration went from $0.397 \mathrm{mS} / \mathrm{cm}$ to $76 \mathrm{mS} / \mathrm{cm}$. The sensor was formed by a toroidal coil and a solenoidal coil. The toroid was powered by a sinewave of $8 \mathrm{~V}$ peak to peak. Some applications of those sensors are presented in [19]. However, even that those sensors presented good calibration and their measuring ranges fit our needs, an optimal encapsulation was not included in the design. The sensor that covers the needs of the aquaculture industry must accomplish some items and the majority of them should be isolated. One of our multiple objectives for this paper is to create a salinity sensor. In order to accomplish this objective, we will design the coils, calibrate the sensor and obtain a good encapsulation system. The encapsulation system must not affect the sensor as it is shown in [36, 37].

In our design, we present a robust and appropriate way to isolate the salinity sensor. There are different prototypes of salinity sensors in previous published works, but none of them has been isolated properly.

\section{Data gathering systems}

In this subsection different data gathering systems are presented. There are several authors who are currently working on technologies aimed to reduce the energy waste. In [38], we can see a classification of these techniques presented by O. Diallo et al. When an alternative data gathering system is proposed, it is necessary to evaluate its effectiveness and to do it, many tests are required. The evaluation of a WSN consumes high amount of resources and time, even higher than the time spend for developing the systems itself. The performance of laboratory test bench and simulations can help us to avoid the real deployments. In this sense, J. Wang et al. developed an adaptive data gathering scheme based on the introduction of an autoregressive model which used their recent work on the emerged compressive sensing theory [39]. This proposal was able to perform a correlation between the value of the sensed data collected by a node and the data gathered by its neighbors. In this proposed system, the number of measures carried out depended on the variation of the sensed parameter and the degree of required 
accuracy. In addition their model was capable of detecting any reading out of bounds. Their experimental results performed with real data made evident the high effectiveness and efficiency of this proposal. Nevertheless, the packets loss of sensed data make very difficult the data reconstruction by using of the autoregressive model. This implies the system performance degradation.

The first design for large-scale WSNs that applied the compressive sampling theory to data gathering process was presented by C. Luo et al. in [40]. The test bench was performed in a scenario with several nodes. As authors commented, the obtained sensed data showed a clear spatial correlation. The results proved the high efficiency and robustness of the proposed system. These results were due to reduction of global scale communication cost without the needed of having intensive computation systems or complex transmission control systems.

The software and hardware design of MPWNodeZ was presented by R. Morais et al. in [41]. Authors implemented three different energy harvesting techniques in their proposal in order to recharge the batteries. This proposal was designed to sense data from large areas and sends them through a wireless acquisition device based on ZigBee technology. The presented proposal was designed to develop precision viticulture in vineyards placed in the Demarcated Region of Douro. Two different tests were carried out. The first one was carried out in the laboratory while the second test was performed in a real field of vineyards. The results show the good performance of their proposal. It could be adapted to precision viticulture.

Our proposal presents some advantages over the current ones. First, our devices work under the IEEE 802.11g standard which reduces the implementation costs. Moreover it offers larger coverage area, thus the number of devices needed to cover an area will be low. The selected device presents a small size which is perfect for environmental and rural monitoring. Although the use of the IEEE 802.11 wireless technology supposes higher energy consumption, the selected device has one of the smallest energy requirements of the commercial devices.

\section{SYSTEM DESCRIPTION AND IMPLEMENTATION}

Our goal is to develop and implement a system that should be able to monitor the salinity changes in an area close to the water pipe and in the tanks. It also should be able to prepare a mix of water to feed the aquaculture facilities by selecting water from two pipes to obtain the desired salinity in each group of tanks. As Fig. 1 shows, the system is based on a WSN deployed around the two water intake pipes. Other sensors will be installed in the aquaculture facility to take information about salinity level. The system will allow the manager of the aquaculture facility to decide the water salinity values required according to the needs. The proposed WSN is composed by nodes connected wirelessly with different technologies. The nodes that monitor the water input pipes are connected to the gateway with WiFi connection. By the other side, the nodes in the facility are connected by Bluetooth, because this technology consumes less energy than WiFi.

The system has two pipes that permit getting water from different places. A common problem in some facilities is the temporal downgrade of the available water quality (related to meteorological alterations, pollution or underwater discharge) and its harmful effects on the fish. However, by having two pipes this problem is reduced. It is possible to select the water from one of the pipes or mix water from both pipes.

The proposed system also includes the water level sensor. This sensor is used to detect the loss of water in one tank. There are different issues that can cause a loss of water in a tank such as the obstruction of the pipes, leakages and even water evaporation. The decrease of water level has several problems associated. The most important one is the reduction of the available oxygen for the fishes, which can cause the dead of the animals. The reduction of spaces is another problem linked to the decrease of water level. It can cause dangerous behaviors such as fish attacks and stress. Finally, the loss of water due to the evaporation can increase the salinity level of the remaining water. 


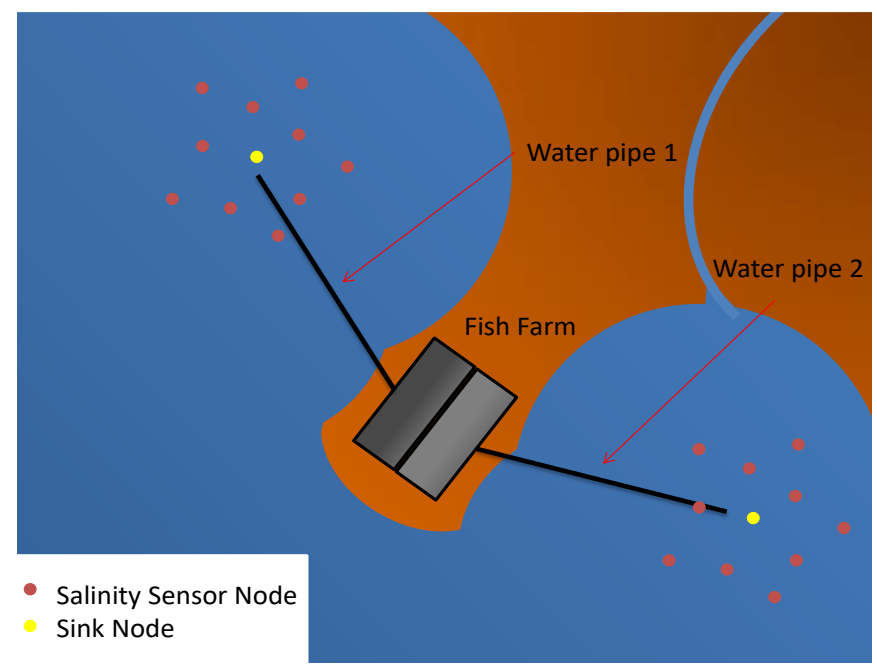

Fig. 1. Fish farm in land

\section{A. Salinity Sensor}

In this subsection, the salinity sensor of our system is presented. The salinity sensor employed in our system is based on the salinity sensor developed by us in [42] with some improvements. They include the development of a waterproof encapsulation and the consideration that the water volume used for calibration is lower that the volume of magnetic field. The sensor is isolated from the environment. As we can see in [36], all the attempts to isolate the sensor using epoxy material failed. Then, we developed two new alternatives, the first one is to use a silicone based insulating pain. The second is to use two PVC tubes to contain the coils inside as it is shown in Fig. 2.

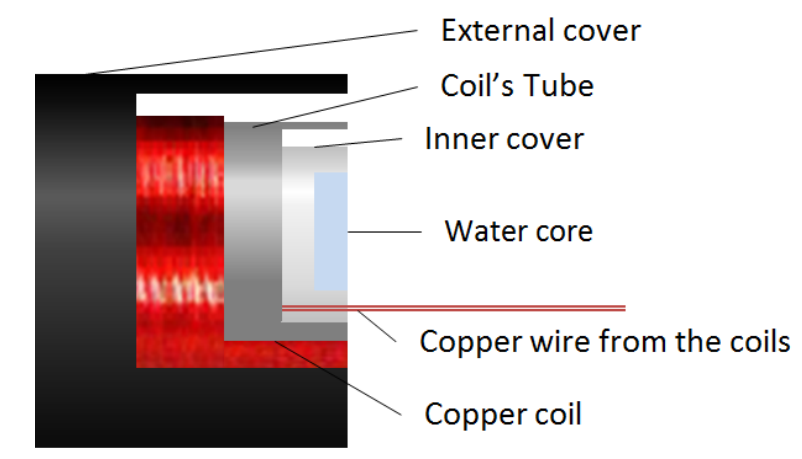

Fig. 2. Design on PVC coverage.

Different coils were used for the isolation test. Due to the experiences shown in [36], we do not use the sensing coil to avoid possible damages, so other prototypes were used. Three different coils were created for this purpose. The coils had 20 spires in powered coil and 40 in the induced coil. The inner diameter of the tube was $1.1 \mathrm{~cm}$ and the external diameter was $1.2 \mathrm{~cm}$. The length of the tube with the coils was $4.2 \mathrm{~cm}$.

The coil with the waterproof painting was left during 24 hours to dry up before the test. The same period of time was applied to the coil with the PVC protection because some silicon was used. Fig. 3 shows the different steps to be performed in order to isolate the coils from water. To make the coil, the first step is to select the support to wind up the copper wire (Fig. 3-c). After that, we put a new PVC tube that covers both coils. We also cover the top and bottom of the coil in order to avoid any contact with the water. To do so, we use a circular piece of plastic that is glued with thermal silicone (Fig. 3-b). Finally, we apply a layer of waterproof paint to seal any pore that may cause water filtration problems (Fig. 3-a). 


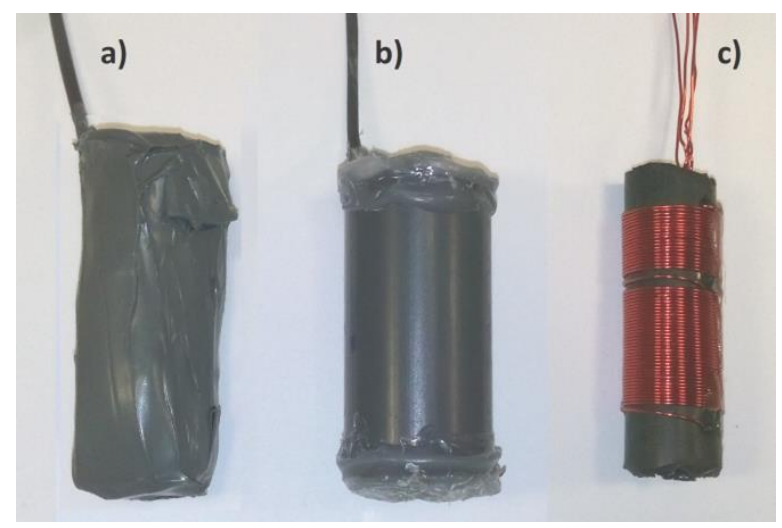

Fig. 3. Sensors with and without isolating cover

In order to evaluate the effects of the waterproof protections on the sensing capacity of the coil, a study is carried out to compare the induced magnetic field. In the case of the coil with waterproof painting the induced magnetic field was almost null. A possible interaction between the varnish and the painting can cause a drastic reduction of the induced magnetic field. The study was done with a saline sample of 43 $\mathrm{mS} / \mathrm{cm}$. We took measures from $1000 \mathrm{kHz}$ to $2000 \mathrm{kHz}$. This data is shown in Table I.

TABLE I. COMPARATIVE BETWEEN ISOLATED PROTOTYPES

\begin{tabular}{|l|c|c|}
\hline \multirow{2}{*}{ Freq. (kHz) } & \multicolumn{2}{|c|}{ Vout (V) } \\
\cline { 2 - 3 } & PVC Waterproof & No waterproof \\
\hline 2000 & 2.2 & 2.4 \\
\hline 1800 & 1.45 & 1.8 \\
\hline 1600 & 0.78 & 1.12 \\
\hline 1500 & 0.63 & 1.01 \\
\hline 1400 & 0.54 & 0.81 \\
\hline 1300 & 0.43 & 0.55 \\
\hline 1100 & 0.28 & 0.39 \\
\hline 1000 & 0.31 & 0.38 \\
\hline
\end{tabular}

The data shown in Table I evidence that the extra PVC tubes for isolating the coil (inner and external cover in Fig. 2) and the air between the covers cause a reduction on the induced magnetic field. This reduction can be expressed as a function of the magnetic field obtained without the PVC tubes. Using the data from Table I and the Analytical software (Eureqa) [43], we obtain (Eq. 1). $V_{\text {iso }}$ represents the output voltage in the isolated coil and $V_{\text {no }}$ iso represents the output voltage on the coil without extra PVC tubes. This equation is used to correct the calibration performed in [44] taking into account the attenuation effect of the plastic coverage.

$$
V_{\text {iso }}(V)=-3.671-\frac{24.375}{V_{n o_{\text {iso }}}(V)-6.553}
$$

Other consideration that must be taken into account is that the generated magnetic field can exceed the volume of the calibration samples. In [37], we calculated the size of the generated magnetic field by the coil. Using the equations of [37] we can correct the obtained output voltage. The loss of the output voltage is $3.2 \%$ for that volume of water. This increase must be applied to the calibration in order to use the sensor in the aquaculture environment, where the amount of water is high enough to contain all the magnetic field. The calibration presented in [42] can be corrected considering Eq. 1 and the 3.2\% of voltage loss, thus it is obtained Eq. 2. Eq. 2 shows the real correlation between the output voltage, from an isolated coil in Volts, and the water conductivity $(\mathrm{mS} / \mathrm{cm})$ in a real scenario. 


$$
V_{\text {iso }}(V)=\left(-3.671-\frac{24.375}{0.5084 \times C\left(\frac{m S}{c m}\right)^{0.4004}-6.553}\right) / 1.032
$$

The equation that will be introduced at the server side to calculate the conductivity using the value of output voltage of the sensor is Eq. 3:

$$
C\left(\frac{m S}{c m}\right)=\sqrt[0.4004]{22.7108-\frac{24.375}{0.524 \times V_{\text {iso }}(V)+1.867}}
$$

\section{B. $\quad$ Wireless module}

In this subsection, the used wireless module is described. Our smart system for monitoring several parameters in aquaculture tanks is based on a wireless module named FlyPort manufactured by openPICUS. The openPICUS Flyport module can be powered by batteries (between 3.3 and 5V). The module is composed by a Certified WiFi Transceiver 802.11g Microchip MRF24WG0MB module. It also contains a 16 bits low power microcontroller. In this case, our module uses a Microchip PIC24FJ256 Processor, with 256K Flash, 16K Ram and able to execute 16MIPS at 32Mhz.

In comparison with some other existing wireless modules, the openPICUS FlyPort is characterized by its versatility in programming and wide application range mainly focused on the Internet of Things (IoT). The node consists of two parts, i.e., the WiFi module which contains the transceiver, the processor the different input/output, buses, etc., and the programmer board named USB Nest.

\section{C. $\quad$ Sensor to measure water level}

The water level sensor proposed for the system is shown in this subsection. GP2Y0A02YK0F (See Fig. 4) is a distance measuring sensor unit manufactured by SHARP. It is composed of an integrated combination of a position sensitive detector, an infrared emitting diode and a signal processing circuit. According to its features, the reflectivity of the object, the environmental temperature and the operating duration are not influenced by the distance detection because this device uses the triangulation method. It is able to measure from 20 to $150 \mathrm{~cm}$. It offers an analog output, where the voltage is related to the detected distance.

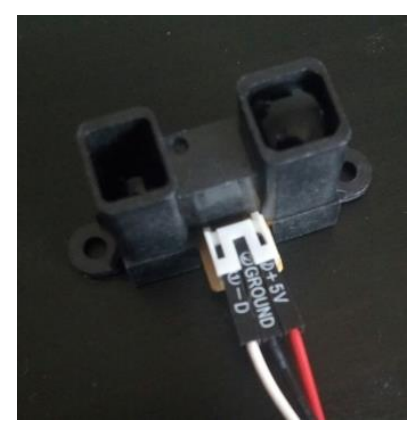

Fig. 4 Distance measuring sensor unit

This sensor is used to detect the water level in fish tanks (See Fig. 5). It is placed at $20 \mathrm{~cm}$ over the water. We have established two thresholds, i.e., the highest level (which corresponds to the regular level) and a lowest level (which corresponds to the critical level). Our fish tanks are continuously monitored in order to ensure that the water maintains the correct water level. 


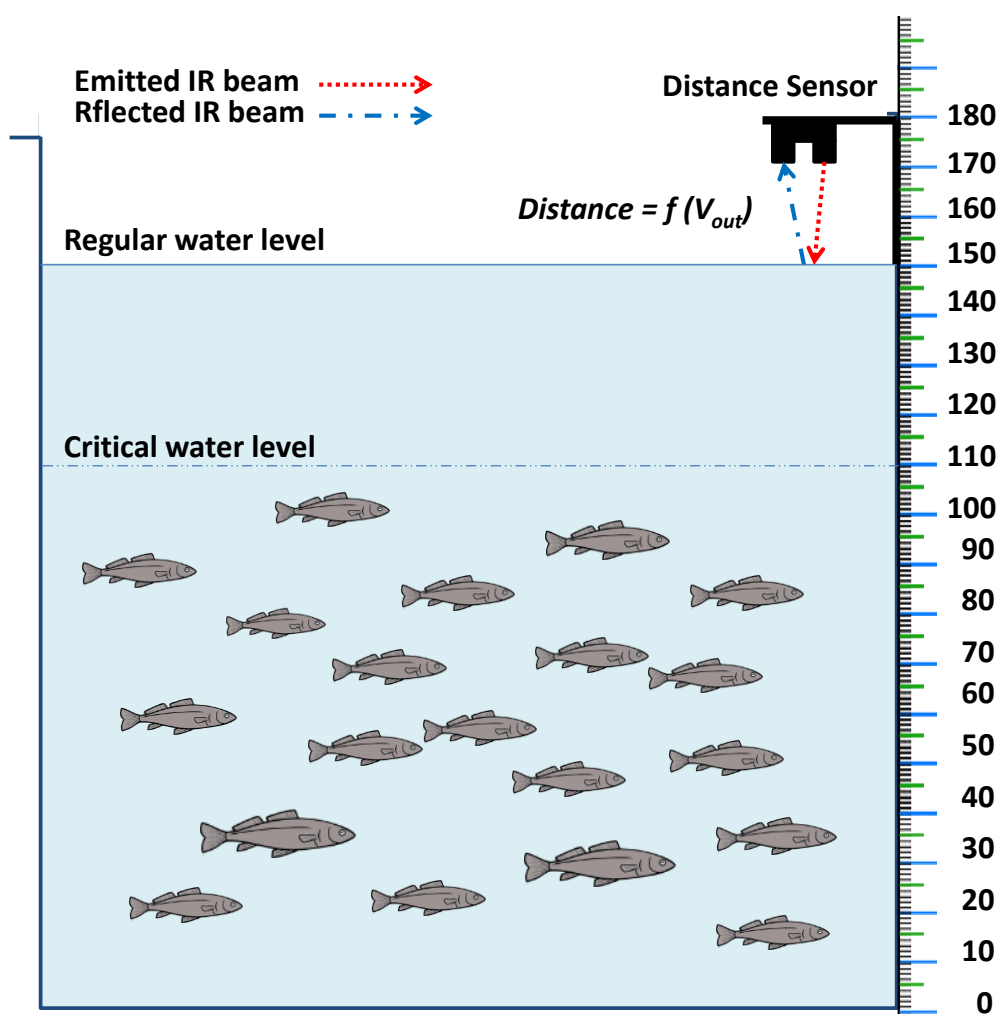

Fig. 5 Distance sensor position in fish tanks

Finally, to understand and model the behavior of this sensor, we have checked the value of the output voltage as a function of the object distance. Fig. 6 shows the results, which is very easy to be modeled by an equation.

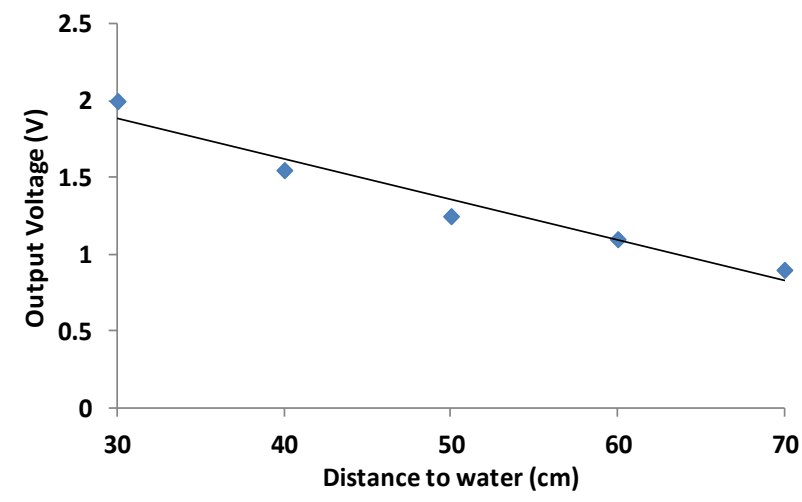

Fig. 6 Distance to water vs. output voltage.

Eq. 4 models the behavior given in Fig. 6 with a $\mathrm{R}^{2}=0.9528$.

$$
\operatorname{Vout}(\mathrm{V})=-0.0265 \times \operatorname{Distance}(\mathrm{cm})+2.685
$$

\section{Signal generation}

The signal generation to feed the salinity sensor is exposed in this subsection. As we explained before, the salinity sensor is composed by two coils; one of them is fed by a signal that records continuous polarity changes. It can be done via square or sine waves. In this case we have selected square signals, due to their simplicity and less need of electronic components. The other coil is induced by the magnetic field which is affected by the salinity.

In order to generate the square signal, we use the same module. The sensor node generates a PWM 
signal. In our case the duty cycle of the square signal is fixed. In order to implement it, the code shown in Algorithm 1 has been used. This code allows to generate the square signal and to gather data from the induced coil. Fig. 7 shows the different stages of our proposed system. The Flyport generates a square wave to feed the first coil, which is placed in the water medium with the second coil. The second coil receives an induced sine wave, which is received by the Flyport as an input measure (sensed value).

Fig. 8 shows the square signal generated by the Flyport using the code of Algorithm 1. It is a square signal at $110.30 \mathrm{kHz}$.

Fig. 9 shows the sine wave obtained at the output of induced coil overlapped with the generated square signal. We can observe that the received signal has a sinus behavior and the amplitude is slightly smaller than the square signal.

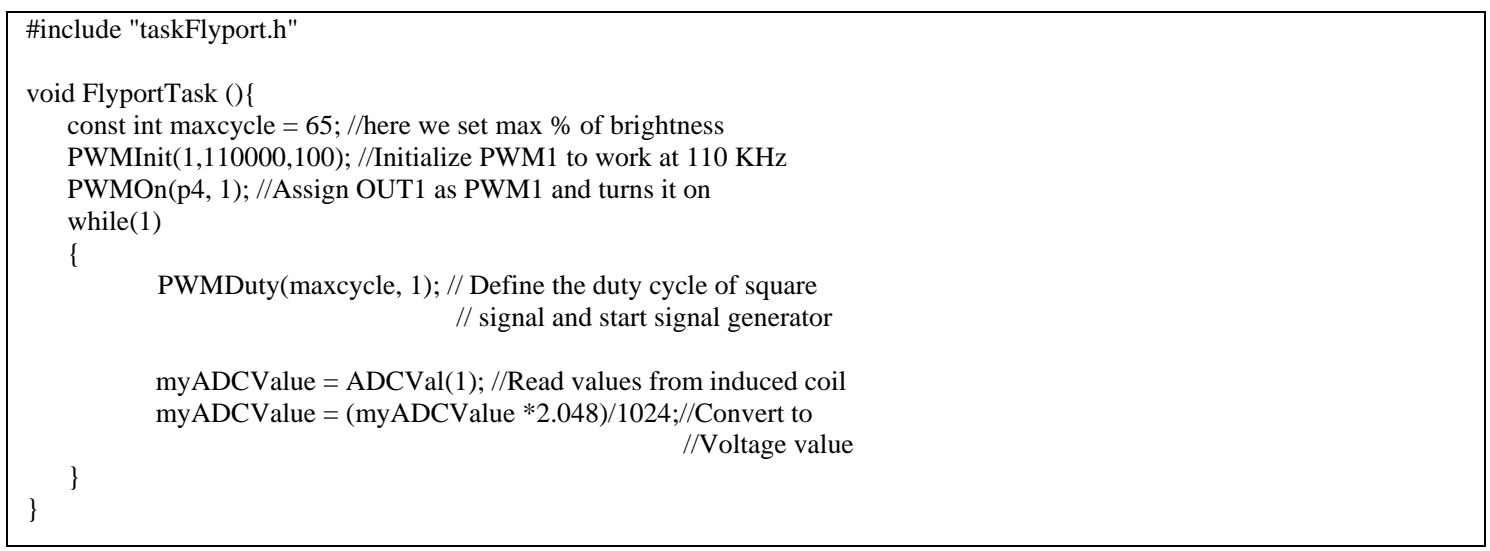

Algorithm 1. Program code for Flyport to create a square signal.

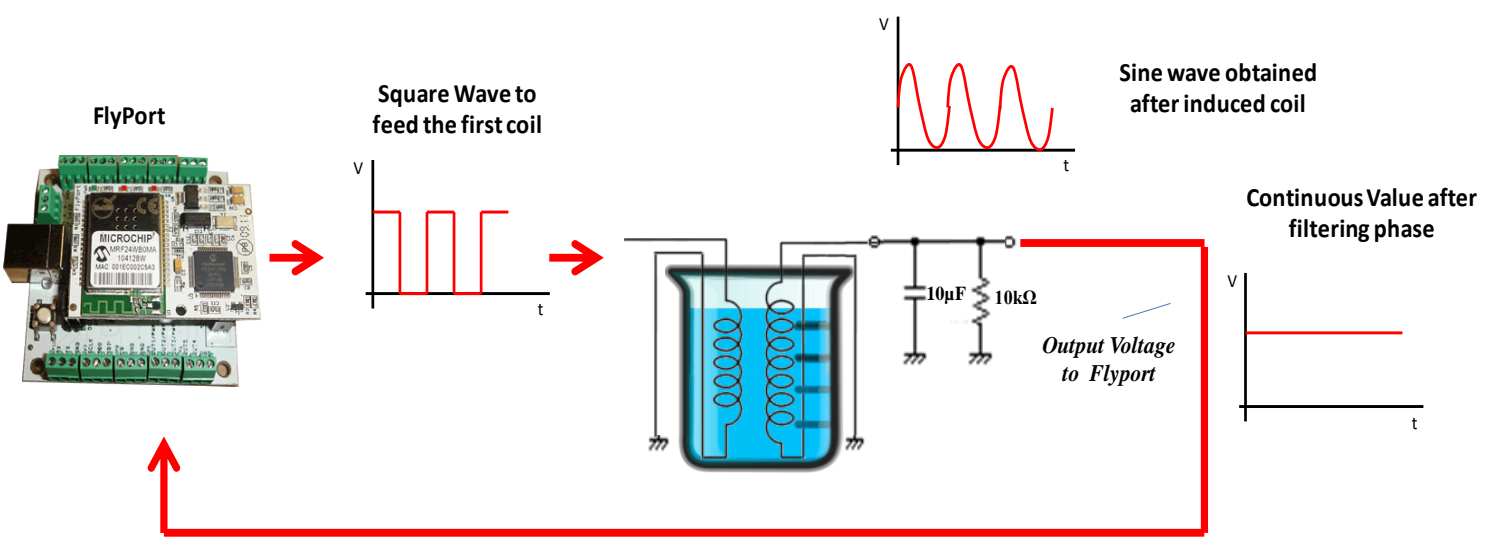

Value gathered by flyPort

Fig. 7 Stages of our proposed system

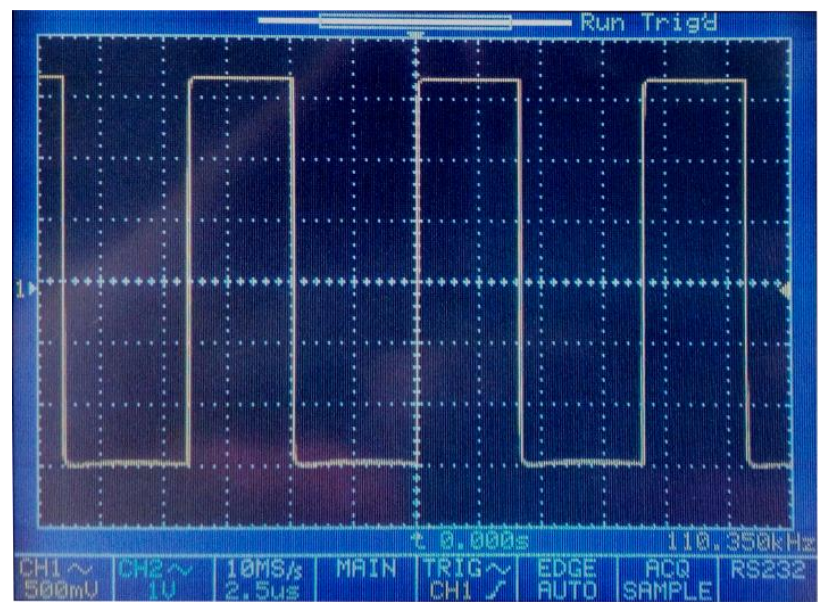

Fig. 8 Square signal generated by the Flyport 


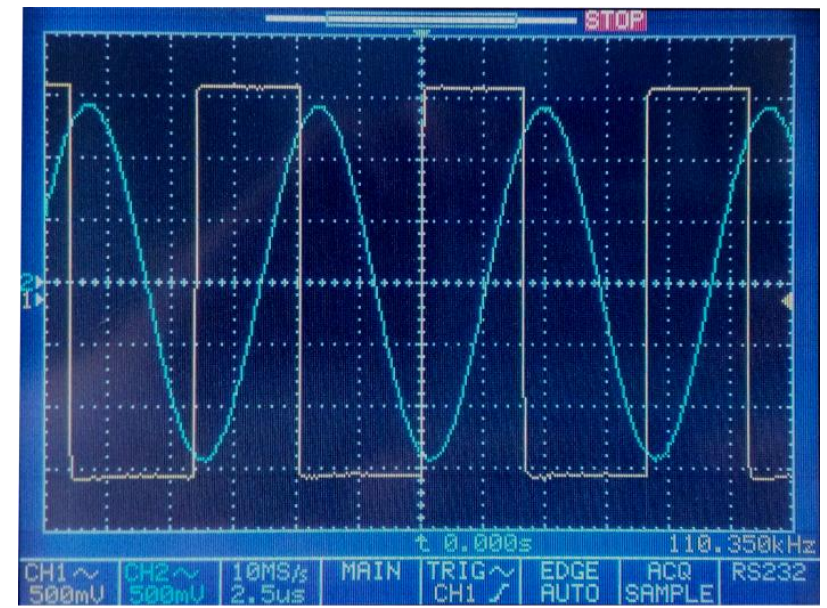

Fig. 9 Sine wave obtained at the output of induced coil overlapped with the generated square signal

\section{E. $\quad$ Server implementationto collect data}

With the goal of gathering and storing data, we have developed a software application that is installed as a service in a server, which requests the node this information and stores it in order to process and take decisions. The application is developed in Java. The information gathered from the sensors is stored in a DB. To implement this, we have selected the connection of devices using sockets. The used of TCP sockets is a widely used and simple method to exchange information. TCP sockets provide a connectionoriented service where data is transferred as a continuous stream. This implies that before transmitting information, a connection must be established between the two devices. In this case, while one of the sockets handles connection requests (server), the other requests the connection (client). After establishing the connection, the server should request data to the client. When the client finishes the data sending, the client sends a Disconnection request packet to the server. After that, the server confirms the disconnection and the server remains in a listening mode while waiting for a new connection. Fig. 10 shows the packet exchange during a connection.

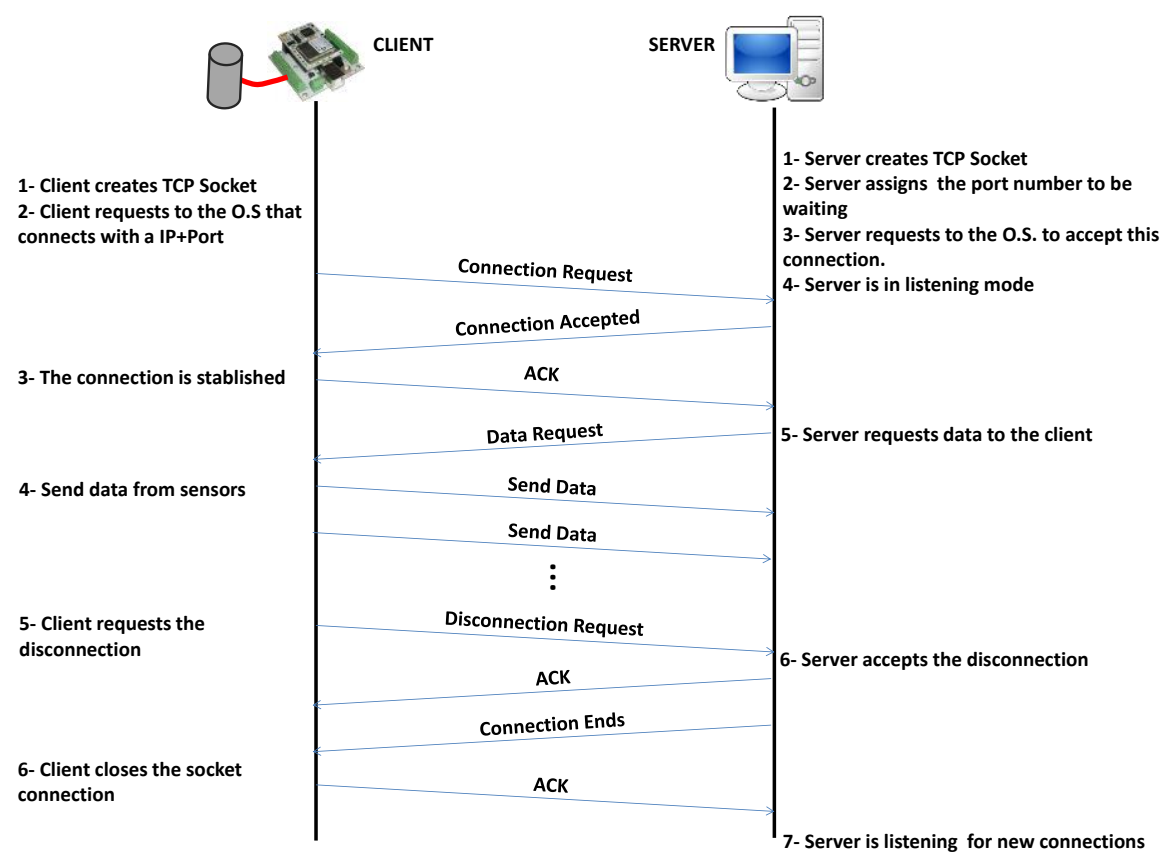

Fig. 10. TPC Socket connection process and packet exchange between server and client

During the process of server configuration the user should specify the number of sensors that will provide data and the port number through which the connection establishment and data exchange will be 
performed. Because the server is implemented using a computer, the application allows to the user choosing the path where the data is going to be saved in a *.csv file. If an additional module is connected to the server, it automatically generates a new file for storing the data provided by this module. Additionally, we need to supply a specific programming for the Flyport module. The program code used in the Flyport module is shown is Algorithm 2. As we can see, the main aim of this algorithm is generating the needed configurations to create a TCP socket but as the role of client. When a node requests for a new connection to the server, it waits to the server reply. When both devices are connected, the server request the data to the node and then the node send the data through the port 8080 .

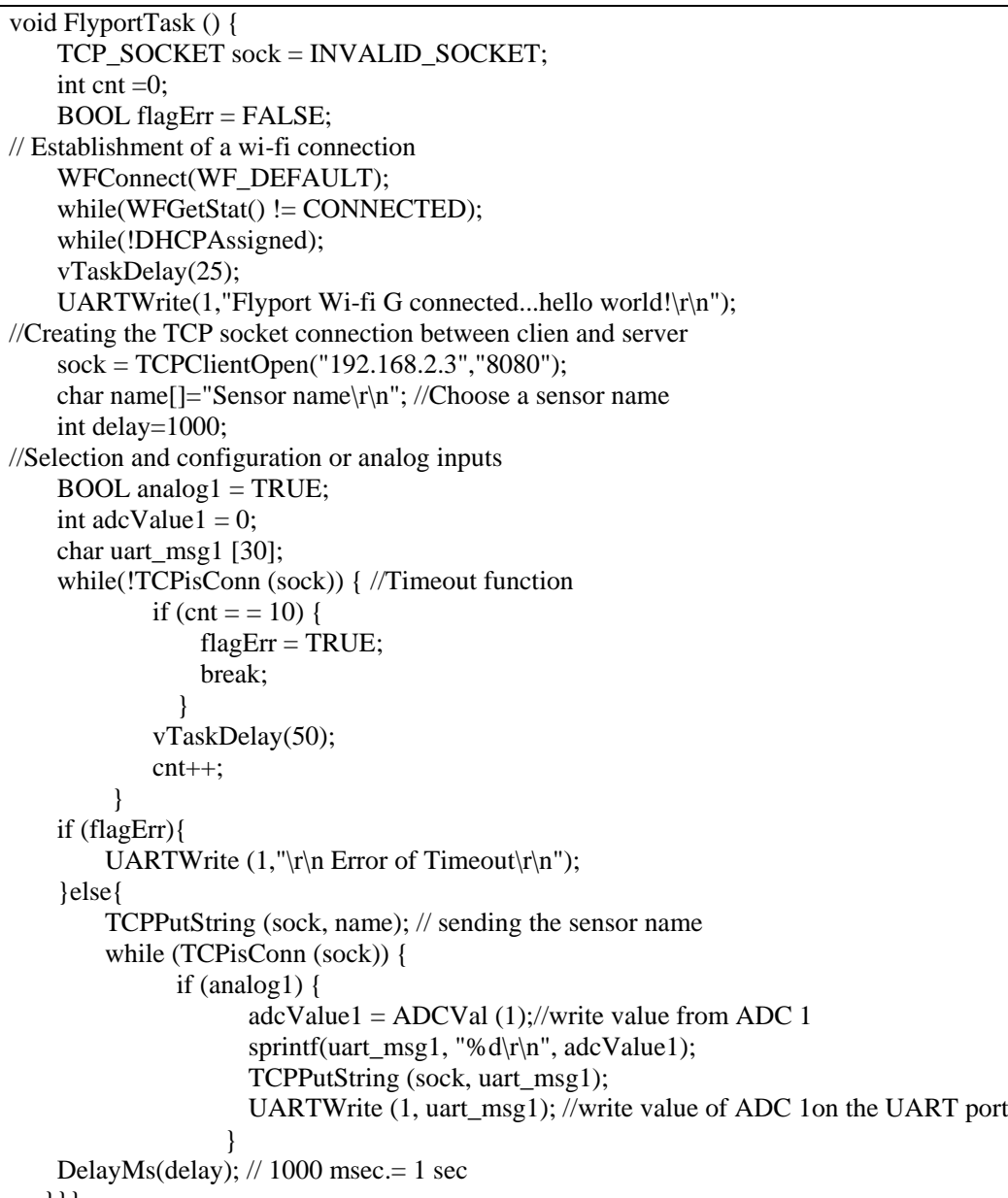

Algorithm 2. Program code for Flyport configuration as Socket Client

\section{AutonOmOUS SYSTEM TO CONTROL AUTOMATICALLY THE WATER SALINITY}

One of the biggest problems when working in aquaculture facilities on land (closed-circuits facilities) is the water evaporation from the fish tanks due to the effect of the sun. This would generate the rise of salinity level that could affect the proper development of the fishes. Another major problem is the loss of water due to an accidental leakage. In this section, we explain the architecture developed and the algorithm needed to control the water levels and salinity concentration in the fish tanks.

As Fig. 1 shows, the facility is located in a land where the water comes from two different areas. In one case, the seawater area is affected by fresh water from a nearby river. This is beneficial for the installation as it allows controlling better the salinity levels when necessary. The control system is shown in Fig. 11. The water can be taken from both sources. This water is propelled towards the intermediate tanks, where strange elements and other fish that enter through the pipe should be removed. This intermediate step can be used to perform other analyzes. Then, the water is brought to the mixers which are responsible of making mixtures of water in the correct proportions.

After this, the water is driven to each group of tanks that should maintain the same salinity level. In order to propel the water between the mixer and fish tanks, water pumps are used. Water valves are used 
to control the access of water to the fish tanks. These elements, as well as the salinity and level sensors are connected and controlled by wireless nodes that receive the control signals required to take the appropriate actions.

This system is adaptable to any type of installation, e.g. open-circuit facilities where water continuously circulates from the sea and other water sources into the tanks of fish and the excess of water is discarded. There are also closed-circuit installations where a fixed volume of water is forced to circulate between the tanks without taking water from external sources.

In order to manage the salinity levels, we have designed an algorithm for the proposed system that allows us to automate the process. Fig. 12 shows the algorithm used by the system to have the appropriate salinity level in the tanks. Initially, the system periodically checks the water level in the tanks using the level sensors (TL). If the water level is normal, the system will check the salinity value of each group of tanks using the salinity sensors (SL). If this value is within the established thresholds, the system will send a message to the controller reporting that the situation is correct. This allows us to keep a history of the recorded values. However, if the system detects that the salinity level is not correct (even though the water level is correct), the system will start to drain some water from the affected tank and the controller will receive an alarm message about the status of the installation. Then, the amount of water needed is calculated. The next step will be to measure the salinity values of the sources from which water will be taken. With these values, it is possible to calculate the volume of water that must be taken from each source, so that the water that leaves the mixer is correct to be sent to the fish tanks. This operation will remain active until the tank reaches the correct water level. Finally, the control system will receive a message about the state of the facilities.

After performing this operation, if the salinity value remains outside the thresholds, this task is repeated as many times as needed to achieve the correct salinity values. Such problems often occur in closedcircuit installations where sometimes water evaporation plays an important role in the proper fish production. Finally, if the system detects a loss of water levels, the same process will be repeated, but considering that the registration problem is not related to an increase/decrease of the water salinity.

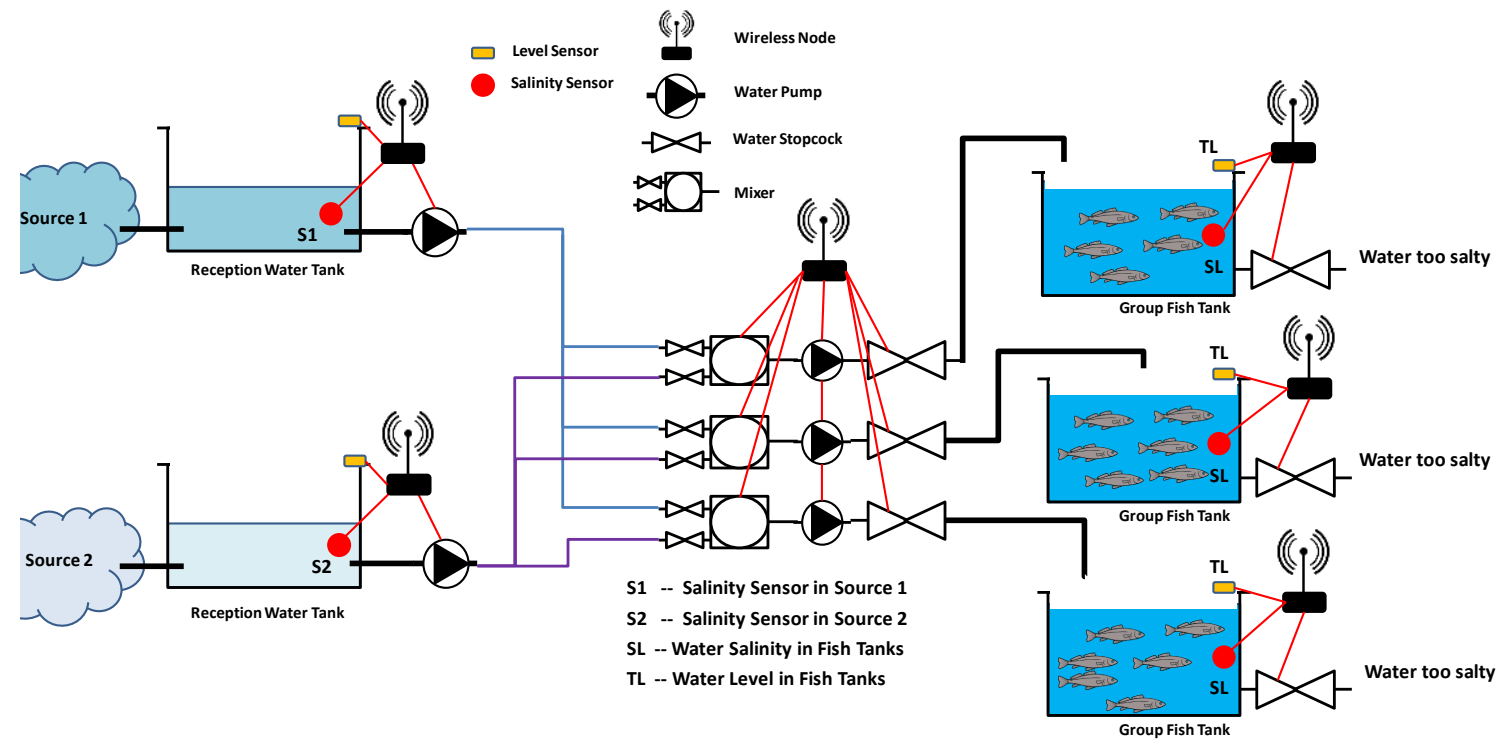

Fig. 11. Architecture and sensors distribution 


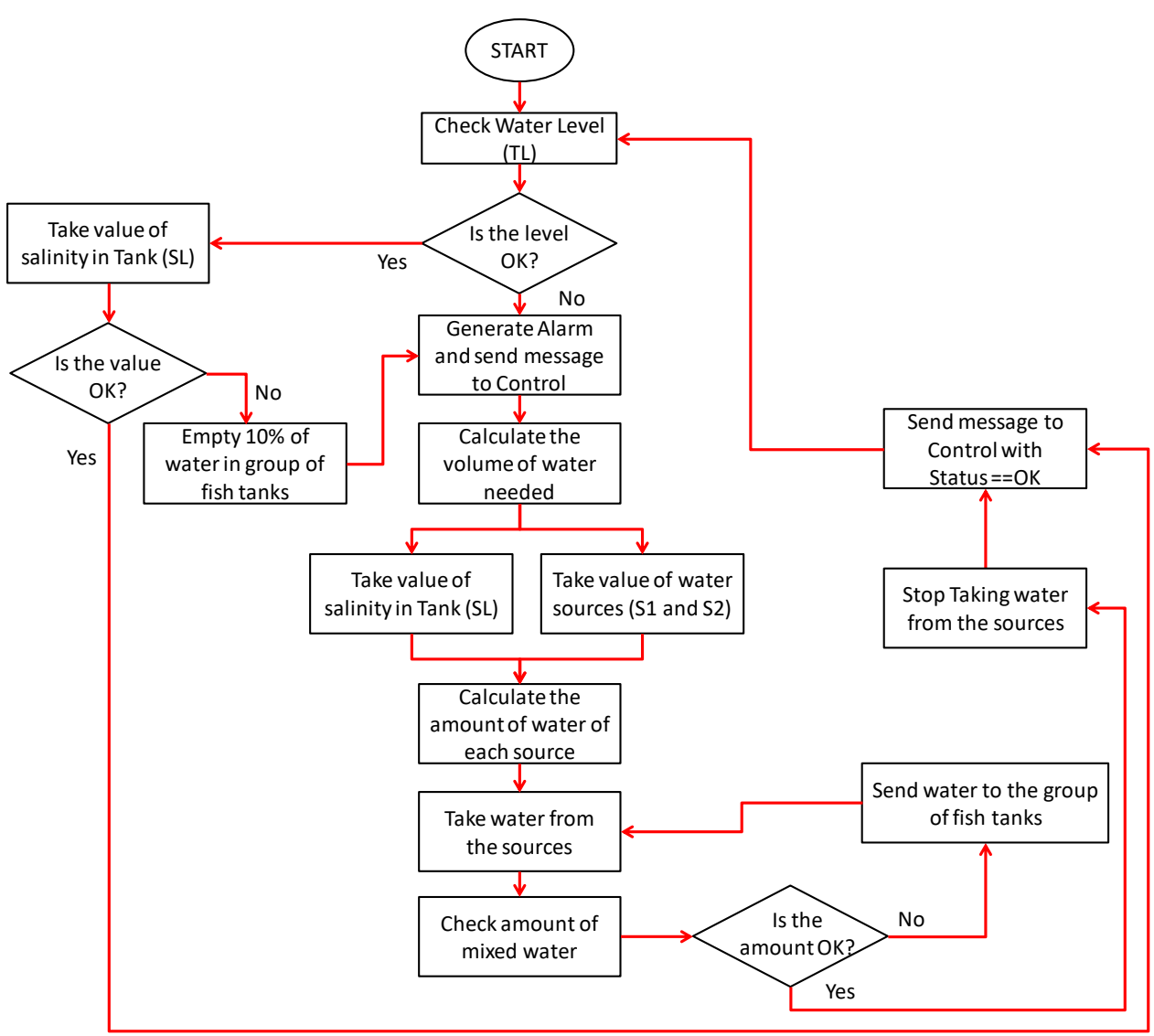

Fig. 12. Flow diagram to control the system

\section{REAL MEASUREMENTS AND NETWORK TEST}

Finally, in order to ensure that our system is able to work properly, we need to check the network performance as well as the response time of the nodes in order to know if the system does not consume too much bandwidth and if it will receive the data with little delay between messages. This section shows the results of the tests about the consumed bandwidth and the delay between received data messages, i.e., the time between TCP packets delivering data from the sensors. It is measured at the server side.

Fig. 13 shows the scenario used in our tests. In this case, we have tested the consumed bandwidth as a function of the kind of device sending the data and the nature of this input. The test is composed by a server, which is in charge of requesting data and collecting them, and 3 different nodes, i.e., a node that takes measures from a salinity sensor which generates analog dada, a node that collect data from 4 level sensors which generates analog dada and a node that registers the water stopcock status. In this last case, we only check if the water stopcock is open or not through a digital input. 


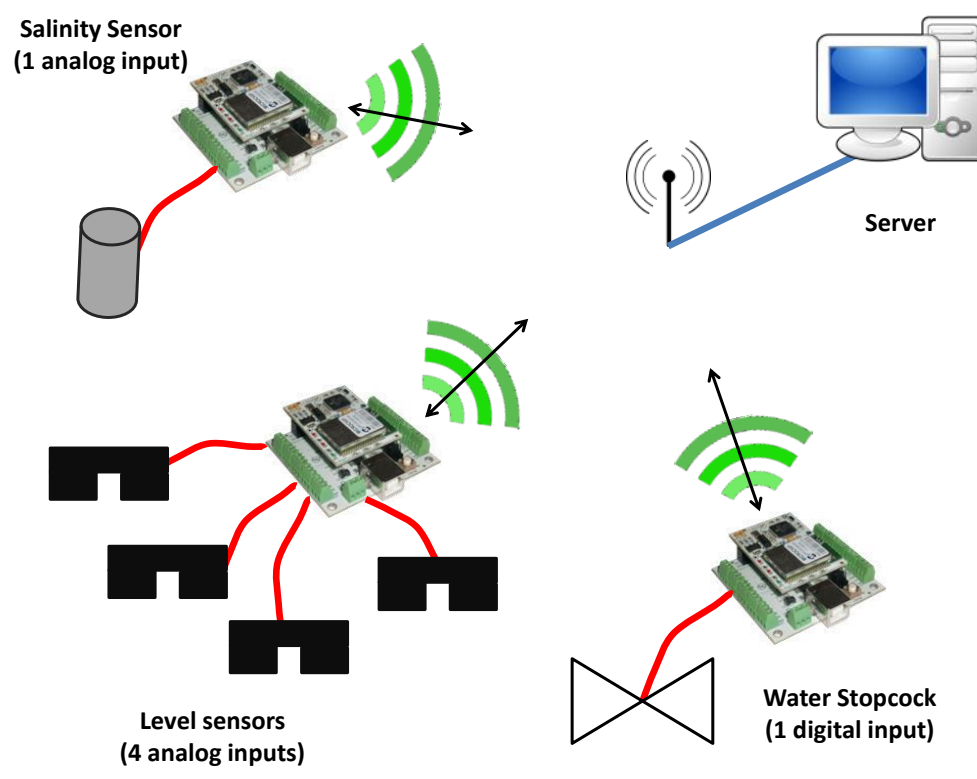

Fig. 13. Scenario used during our test bench

In order to test each node separately, we only transmitted data with one node each time. Fig. 14 shows the consumed bandwidth by a node when sending data from 4 level sensors. Data of these sensors are gathered by the analog inputs. As we can see, the average consumed bandwidth is about 400 Bytes/s. There is a peak about 2100 Bytes/s which could correspond to a retransmission of some lost packets. The consumed bandwidth is quite regular.

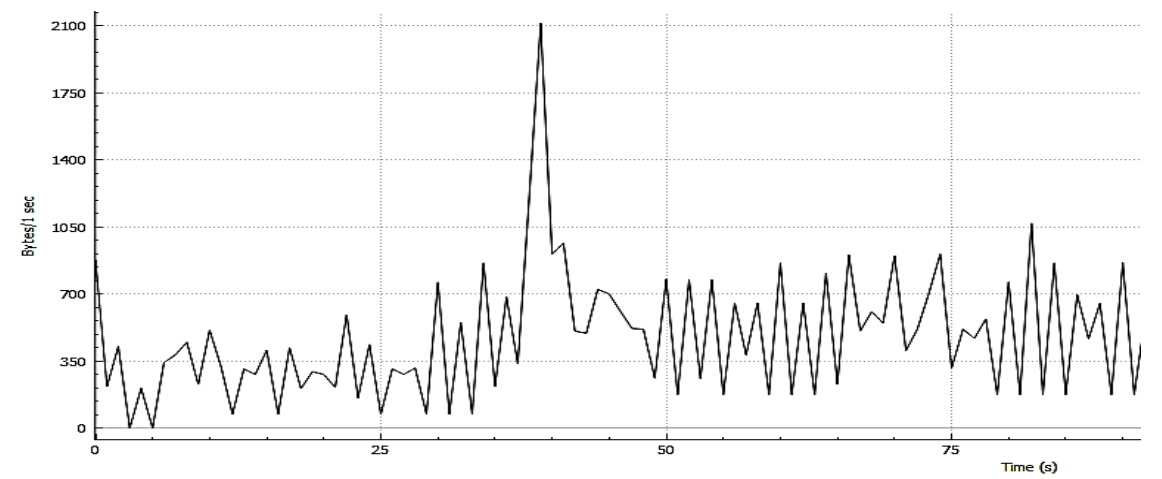

Fig. 14. Consumed bandwidth by a node when sending data from 4 level sensors

Fig. 15 shows the consumed bandwidth by a node when sending data from a salinity sensor. Data of this sensor are gathered by the analog input. In this case, the consumed bandwidth is quite variable. We can observe time periods with an average bandwidth about 600 Bytes/s (at the beginning of the test) and other where the average bandwidth is about 210 Bytes/s. We have also observed some peaks higher than 800 Bytes/s.

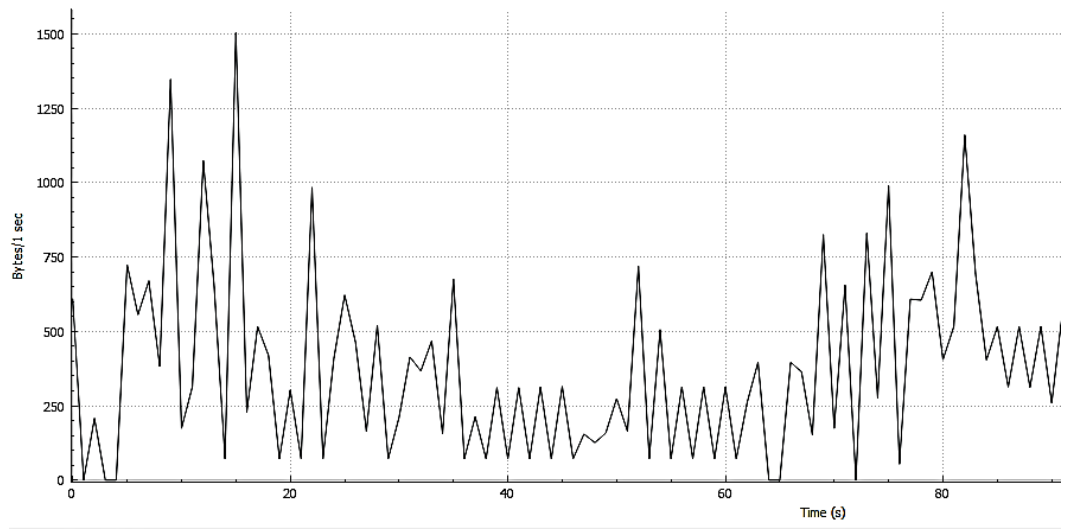

Fig. 15. Consumed bandwidth by a node when sending data from one salinity sensor 
Finally, Fig. 16 shows the consumed bandwidth by a node when taking data from a water stopcock. Data of this sensor is captured through a digital input. In this case, we only take measurements about its status, i.e., if it is open or closed. The behavior, in this case, is quite stable with an average bandwidth of about 400 Bytes/s. There are some few peaks higher than 800 Bytes/s.

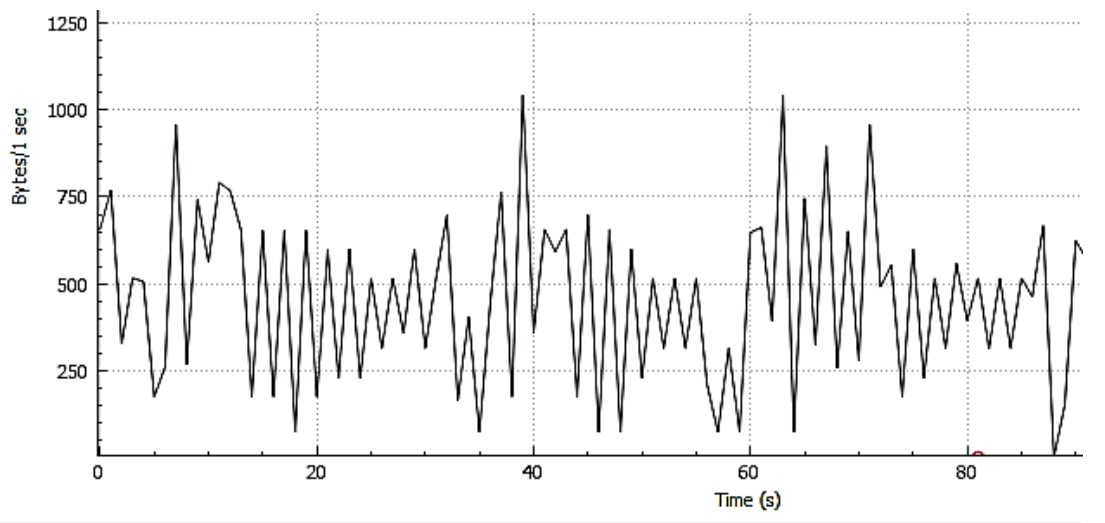

Fig. 16. Consumed bandwidth by a node when sending data from level sensor.

Finally, Fig. 17 shows the delay between TCP messages delivering data from the sensors with the gathered measurements and their status. During the tests, 92 pairs of TPC streams are sent. We can observe that in all cases, the delay from the previous message to the next one seems to follow a periodical behavior. Additionally, the salinity sensor presents higher peaks with values around 1.2 seconds.

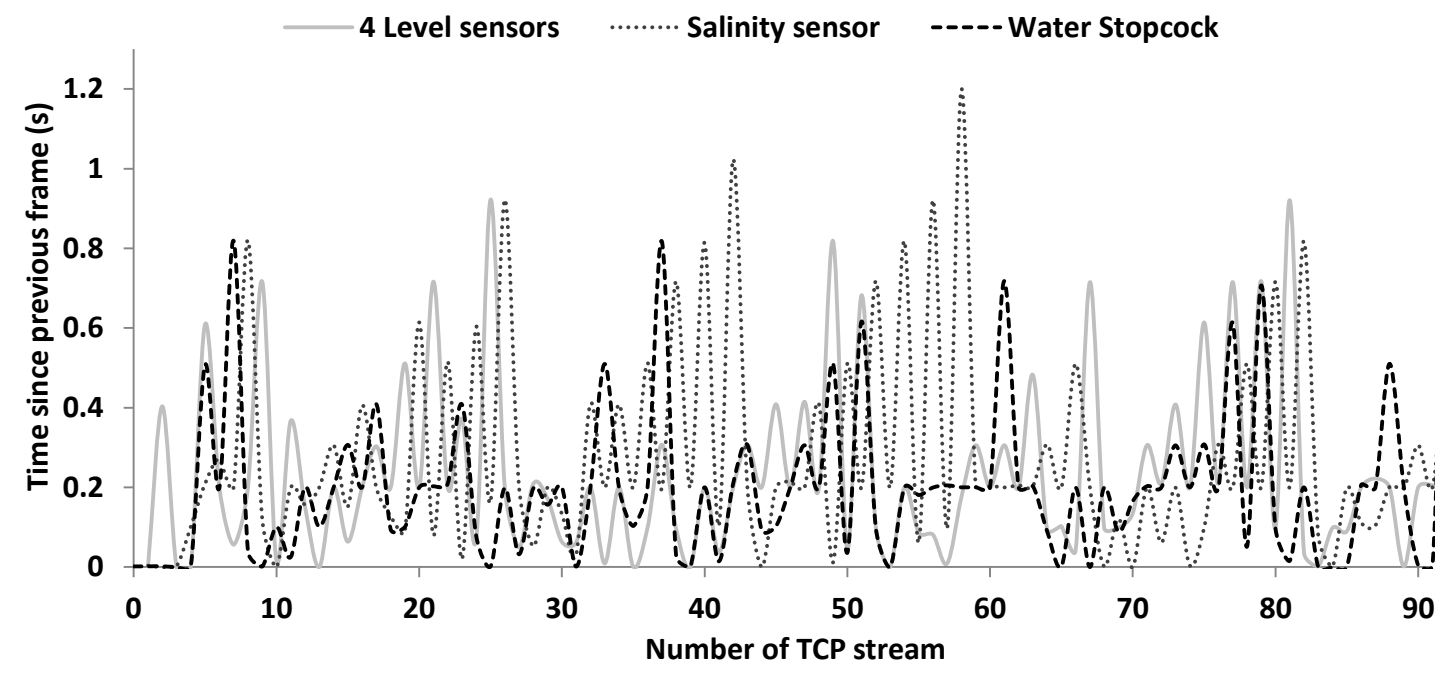

Fig. 17. Delay between TCP streams.

Finally, Fig. 18 shows the histogram to count the number of messages as a function of the delay between TCP streams. 


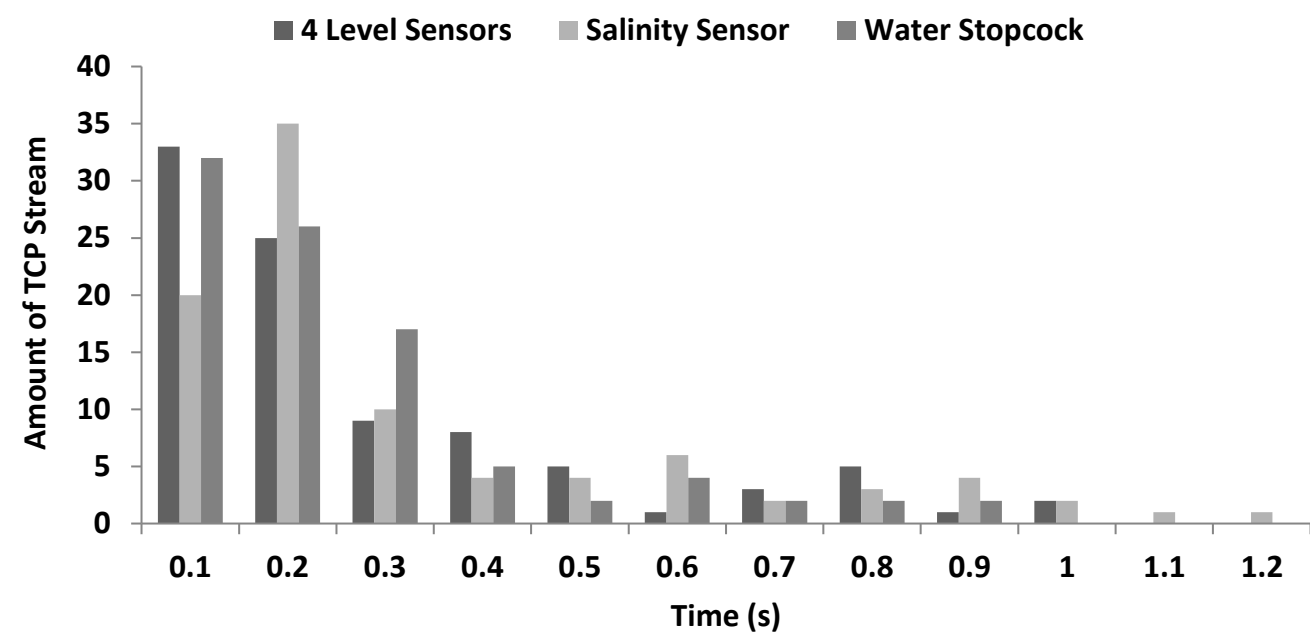

Fig. 18. Histogram of the delay between TCP messages.

It is easy to see that the largest number of packets presented a delay between TCP messages smaller than 0.3 seconds. While level sensors and water stopcock concentrate the many packets in times lower than $0.1 \mathrm{~ms}$, salinity sensor have more amount of packets at $0.2 \mathrm{~ms}$.

From these results we can conclude that our system could be used as a good monitoring system with little delay between the received data.

\section{CONCLUSION AND FUTURE WORK}

In this paper the design of a smart system for data gathering in aquaculture tanks using a WSN and its deployment have been presented. It has been specially designed for fish farms composed by tanks in land which should take water from two different sources.

It is required to control the water salinity in order to control the fish growth. The system allows the facility manager to increase or reduce the fish growth beyond the temperature changes to cover the market demands

The design and deployment of our system included the salinity sensor and the distance sensor description with their calibrations. We have also shown the wireless module operation, its program code to implement the different functions as well as how to store data in a DB. Finally, we have developed and explained an autonomous algorithm which is able to adapt the system operation to the environment conditions and tank status (water level and salinity value). To carry out the test of our system, real samples and real scenario were used. Regarding to the process of taking measurements, the proposed system presents stable values and during the sending data task the consumed bandwidth is low. This fact makes possible the use of a large number of wireless modules to cover a significant area without registering important delays in transmissions.

Our future work will be focused on using more secure methods for establishing the session between server and clients. In our following implementation, we will use SSL sockets. Moreover, we pretend to extend the use of our application for Waspmote and other well-known wireless modules.

The versatility of this server and the rest of the designed system allow the use of other sensors for monitoring different water parameters. Using some other sensor combination, this system could also be used to monitor fish activity in fish farms [44] and to a automate water supplies for precision agriculture adapting some parts of this system.

\section{ACKNOWLEDGMENT}

This work has been partially supported by the Postdoctoral Scholarship "Contratos Postdoctorales UPV 2014 (PAID-10-14)" of the "Universitat Politècnica de València", by the "Programa para la Formación de Personal Investigador-(FPI-2015-S2-884)" of the "Universitat Politècnica de València", by the predoctoral student grant "Ayudas para contratos predoctorales de Formación del Profesorado Universitario FPU (Convocatoria 2014)" Reference: FPU14/02953 by the "Ministerio de Educación, Cultura y Deporte", by Instituto de Telecomunicações, Next Generation Networks and Applications Group (NetGNA), Covilhã Delegation, by the National Funding from the FCT-Fundação para a Ciência e a Tecnologia through the UID/EEA/500008/2013 Project, by the Government of Russian Federation, Grant 074-U01, and by Finep, with resources from Funttel, Grant No. 01.14.0231.00, under the 
Radiocommunication Reference Center (Centro de Referência em Radiocomunicações — CRR) project of the National Institute of Telecommunications (Instituto Nacional de Telecomunicações_-Inatel), Brazil.

\section{REFERENCES}

[1] J. Yick, B. Mukherjee, D. Ghosal, Wireless sensor network survey. Computer networks 2008; 52(12): 22922330.

[2] A.P. Das, S.M. Thampi, Simulation tools for Underwater sensor networks: A survey, Network Protocols and Algorithms 2016; 8(4) :. Doi:10.5296/npa.v8i4.10471

[3] S. EL Mamoune, M. Ezziyyani, J. Lloret, Towards a New Approach for Modelling Interactive Real Time Systems Based on Collaborative Decisions Network, Network Protocols and Algorithms 2015; 7(1) : 42-63.

[4] S. Manel and M. Aref. Optimal load balanced clustering in homogeneous wireless sensor networks. International Journal of Communication Systems, 2016.

[5] L. Haibo, H.E. Minghua, R. Zhiqiang. Time-aware and energy-efficient opportunistic routing with residual energy collection in wireless sensor networks. International Journal of Communication Systems, 2016.

[6] B. Abhijit, R. Sanjay Dhar, K. Sumit. Cognitive radio network with continuous energy-harvesting. International Journal of Communication Systems, 2016.

[7] H.M. Ammari, N. Gomes, M. Jacques, W. Grosky, B. Maxim, D. Yoon, A Survey of Sensor Network Applications and Architectural Components, Ad Hoc and Sensor Wireless Networks 2015, 25 (1-2):1-44

[8] A. Bagchi, A. Madan, A. Premi, S. Sankhla, Hierarchical Neighbor Graphs: A Topology Control Mechanism for Data Collection in Heterogeneous Wireless Sensor Networks, Ad Hoc and Sensor Wireless Networks 2015, 26 (1-4): 171-191.

[9] C. Cambra, S. Sendra, J. Lloret, L. Parra, Ad hoc Network for Emergency Rescue System based on Unmanned Aerial Vehicles, Network Protocols and Algorithms 2015; 7(4) : 72-89

[10] S. Yan, Sensor Networks for Cyber Physical Systems. Ad hoc and Sensor Wireless Networks 2015; 25(3-4) : 241-261

[11] N. Wang, N. Zhang, M. Wang, Wireless sensors in agriculture and food industry-Recent development and future perspective. Computers and electronics in agriculture 2006; 50(1) : 1-14.

[12] G. Werner-Allen, J. Johnson, M. Ruiz, J. Lees, M. Welsh, Monitoring Volcanic Eruptions with a Wireless Sensor Network. Proceedings of IEEE Second European Workshop on Wireless Sensor Networks, Istanbul, Turkey, January 31- February 2 2005; 108-120.

[13] J. Beutel, S. Gruber, A. Hasler, R. Lim, A. Meier, C. Plessl, I. Talzi, L. Thiele, C. Tschudin, M. Woehrle, M. Yuecel, PermaDAQ: A Scientific Instrument for Precision Sensing and Data Recovery in Environmental Extremes. Proceedings of 2009 International Conference on Information Processing in Sensor Networks, San Francisco, USA, April 13-16, 2009; 265-276.

[14] J. Lloret, I. Bosch, S. Sendra, A. Serrano, A wireless sensor network for vineyard monitoring that uses image processing. Sensors $2011 ; \mathbf{1 1}(6)$ : 6165-6196

[15] C. Bajracharya, R. Grodi, D. B. Rawat, Performance analysis of wireless sensor networks for wind turbine monitoring systems, In proc. of the IEEE SoutheastCon 2015, Fort Lauderdale, Florida (USA),April 9-12, 2015; $1-4$.

[16] T.J. Chowdhury, C. Elkin, V. Devabhaktuni, D.B.Rawat, J. Oluoch, Advances on Localization Techniques for Wireless Sensor Networks: A Survey. Computer Networks 2016; 110 (9) : 284-305.

[17] C. Elkin, R. Kumarasiri, D.B. Rawat, V. Devabhaktuni, Localization in wireless sensor networks: A DempsterShafer evidence theoretical approach. Ad Hoc Networks 2017; 54 (-) : 30-41.

[18] L. Parra, S. Sendra, J.M. Jimenez, J. Lloret, Smart system to detect and track pollution in marine environments. Proceedings of 2015 IEEE International Conference on Communication Workshop (ICCW 2015), London, United Kingdom, June 08-12 2015; 1503-1508.

[19] S. Sendra, L. Parra, J. Lloret J.M. Jiménez, Oceanographic Multisensor Buoy Based on Low Cost Sensors for Posidonia Meadows Monitoring in Mediterranean Sea. Journal of Sensors 2015, 2015, Article ID 920168 : 1-23

[20] V.C. Rubio, F.J. Sánchez-Vázquez,J.A. Madrid, Effects of salinity on food intake and macronutrient selection in European sea bass. Physiology \& behavior 2005; 85(3) : 333-339.

[21] R. Laiz-Carrión, S.Sangiao-Alvarellos, J.M. Guzmán, M.P.M. del Río, J.L. Soengas, J.M. Mancera, Growth performance of gilthead sea bream Sparus aurata in different osmotic conditions: implications for osmoregulation and energy metabolism. Aquaculture 2005; 250(3) : 849-861.

[22] L.A. Sampaio, A. Bianchini, Salinity effects on osmoregulation and growth of the euryhaline flounder Paralichthys orbignyanus. Journal of Experimental Marine Biology and Ecology 2002; 269(2) : 187-196.

[23] T.I. Smith, M.R. Denson, L.D. Heyward, W.E. Jenkins, L.M. Carter, Salinity effects on early life stages of southern flounder Paralichthys lethostigma. Journal of the World Aquaculture Society 1999; 30(2) : 236-244.

[24] C.K. Faulk and G.J. Holt, Responses of cobia Rachycentron canadum larvae to abrupt or gradual changes in salinity. Aquaculture 2006; 254(1) : 275-283.

[25] A. Tandler, F.A. Anav, I. Choshniak, The effect of salinity on growth rate, survival and swimbladder inflation in gilthead seabream, Sparus aurata, larvae. Aquaculture 1995; 135(4) : 343-353. 
[26] D.S. Fielder, W. Bardsley, A preliminary study on the effects of salinity on growth and survival of mulloway Argyrosomus japonicus larvae and juveniles. Journal of the World Aquaculture Society 1999; 30(3) : 380-387.

[27] I.P. Saoud, S. Kreydiyyeh, A. Chalfoun, M. Fakih, Influence of salinity on survival, growth, plasma osmolality and gill $\mathrm{Na}+-\mathrm{K}+-\mathrm{ATPase}$ activity in the rabbitfish Siganus rivulatus. Journal of Experimental Marine Biology and Ecology 2007; 348(1) : 183-190.

[28] M.Y. Tsuzuki, J.K. Sugai, J.C. Maciel, C.J. Francisco, V.R. Cerqueira, Survival, growth and digestive enzyme activity of juveniles of the fat snook (Centropomus parallelus) reared at different salinities. Aquaculture 2007; 271(1) : 319-325.

[29] O. Postolache, P.S. Girão, J.M.D. Pereira, Water Quality Monitoring and Associated Distributed Measurement Systems: An Overview. WATER QUALITY MONITORING AND ASSESSMENT. 2012 (-) : 25-65.

[30] Y. Zhao, B. Zhang, Y. Liao, Experimental research and analysis of salinity measurement based on optical techniques. Sensors and Actuators B: Chemical 2003; 92(3) : 331-336.

[31] J. Wang , B. Chen, Experimental research of optical fiber sensor for salinity measurement. Sensors and Actuators A: Physical 2012; 184 (-): 53-56.

[32] J.R. Guzman-Sepulveda, V.I. Ruiz-Perez, M. Torres-Cisneros, J.J. Sanchez-Mondragon, D.A. May-Arrioja, Fiber optic sensor for high-sensitivity salinity measurement. IEEE Photonics Technology Letters 2013; 25(23) : 2323-2326.

[33] K. Striggow, R. Dankert, The exact theory of inductive conductivity sensors for oceanographic application. IEEE Journal of Oceanic Engineering 1985; 10(2):175-179.

[34] T.T. Pham, D. Burnett, L. Handugan, D. Frashure, J. Chen, L. Bushnell, Low Cost, Data-Logging Salinity Sensor. Proceedings of IEEE OCEANS 2007, Scotland, United Kingdom, September 18-21 2007; 1-5

[35] T.T. Pham, T. Green, J. Chen, P. Truong, A. Vaidya, L. Bushnell, A salinity sensor system for estuary studies. Proceedings of IEEE OCEANS 2008 Quebec, Canada September 15-18 2008; 1-6.

[36] R.T. Wood, A. Bannazadeh, N.Q. Nguyen, L.G. Bushnell, A salinity sensor for long-term data collection in estuary studies. Proceedings of IEEE OCEANS 2010, Sydney, Australia, May $24-27$ 2010; 1-6.

[37] L. Parra, V. Ortuño, S. Sendra, J. Lloret, Low-Cost Conductivity Sensor Based on Two Coils. Proceedings of First International Conference on Computational Science and Engineering (CSE'13), Valencia, Spain, August 6-8, 2013; 107-112.

[38] O. Diallo, J.J.P.C. Rodrigues, M. Sene, J. Lloret, Distributed Database Management Techniques for Wireless Sensor Networks. IEEE Transactions on Parallel and Distributed Systems 2013; 26(2): 604-620.

[39] J. Wang, S. Tang, B. Yin, X.Y. Li, Data Gathering in Wireless Sensor Networks Through Intelligent Compressive Sensing. Proceedings of the 31st Annual IEEE International Conference on Computer Communications (IEEE INFOCOM 2012), Orlando, USA, March 25-30 2012; 603-611.

[40] C. Luo, F. Wu, J. Sun, C.W. Chen, Compressive Data Gathering for Large-Scale Wireless Sensor Networks. Proceedings of the 15th annual International conference on Mobile computing and networking (ACM 2009), Beijin, China, September 20-25 2009; 145-156.

[41] R. Morais, M.A. Fernandes, S.G. Matos, C. Serôdio, P.J.S.C. Ferreira, M.J.S.C Reis, A ZigBee multi-powered wireless acquisition device for remote sensing applications in precision viticulture. Computers and electronics in agriculture 2008; 62(2) : 94-106.

[42] L. Parra, E. Karampelas, S. Sendra, J. Lloret, J.J. Rodrigues, Design and deployment of a smart system for data gathering in estuaries using wireless sensor networks. Proceedings of the 2015 International Conference on Computer, Information and Telecommunication Systems, Gijon, Spain, July 15-17 2015, 1-5.

[43] Eureqa Formulizer software. Available at: http://www.nutonian.com/products/eureqa/[Last access: January 3, 2017]

[44] J. Lloret, S. Sendra, M. Garcia, G. Lloret, Group-based underwater wireless sensor network for marine fish farms. Proceedings of the 2011 IEEE Global Communications Conference, Houston, TX, December 5-9 2011; 115-119. 